\title{
Self-point defect trapping responsible for radiation swelling reduction in V-Ti alloys
}

\author{
A.O. Boev $^{\text {a, }}{ }^{*}$ I.V. Nelasov ${ }^{\text {b }}$, A.G. Lipnitskii ${ }^{\text {c }}$, A.I. Kartamyshev ${ }^{\text {d }}$, D.A. Aksyonov ${ }^{\text {a }}$ \\ ${ }^{a}$ Skolkovo Institute of Science and Technology, 121205, Moscow, Russia \\ ${ }^{\mathrm{b}}$ Institute of Problems of Chemical Physics (IPCP), Russian Academy of Sciences, Chernogolovka, Moscow, 142432, Russia \\ ${ }^{\mathrm{c}}$ Belgorod State University, 308015, Belgorod, Russia \\ ${ }^{\mathrm{d}}$ Ton Duc Thang University, Ho Chi Minh City, Viet Nam
}

\section{A R T I C L E I N F O}

Communicated by Y.E. Lozovik

\section{Keywords:}

Molecular dynamics

Diffusion

Vanadium-titanium

Defect complexes

\begin{abstract}
A B S T R A C T
The very low swelling of $\mathrm{V}-\mathrm{Ti}$ alloys under neutron irradiation is a poorly understood phenomenon. To uncover the underlying mechanism of swelling reduction, we performed density functional theory and molecular dynamics simulations with a V-4\% Ti random alloy with interatomic potentials. We find that titanium solutes efficiently trap vacancies and self-interstitial atoms. Exceed vacancies conserve their individuality, forming a variety of complexes with Ti solutes compared to their association into a dislocation loop in pure V. Randomly distributed Ti-vacancy complexes are much more appropriate for efficient point defect recombination as opposed to the localized dislocation loop formed in pure V.
\end{abstract}

Alloying of $\mathrm{V}$ with $\mathrm{Ti}$ is one of the most efficient approaches to reduce radiation-induced swelling, making $\mathrm{V}-\mathrm{Ti}$ alloys safe for operation under neutron irradiation at $650-1000 \mathrm{~K}$ [1-3]. For example, compared to pure $\mathrm{V}$, the addition of 4.9 at.\% Ti reduces swelling in the alloy by more than two orders of magnitude [4], and no other element can provide comparable results. The mechanism of this reduction was proposed in our previous study, where existence of complex vacancy-Ti defects in a V-Ti system was discovered using density functional theory (DFT) [5]. For example, a Ti-Vac-Ti complex has a binding energy of $0.80 \mathrm{eV}$, which is significantly higher than that for a simple Ti-Vac pair $(0.37 \mathrm{eV})$. Using a simple analytic model of void swelling, we estimated that Ti-Vac-Ti complexes can reduce radiation swelling by two orders of magnitude in the working temperature range of $\mathrm{V}-\mathrm{Ti}$ alloys due to vacancy trapping.

The estimation, however, neglects that in reality a range of defect complexes with different trapping energies is acting at the same time. Moreover, the analytic model takes into account only the increase of activation energy for diffusion, while alloying with titanium can also change the pre-exponential factor. The larger scale effect of vacancy trapping on microstructure is also not clear, though it is required for comparison with experimental results. Finally, the influence of trapping on capture efficiency of self-interstitial atoms is not established yet.

To address these issues in this letter we adopt a molecular dynamics
(MD) simulation using classical V-Ti potentials, which allowed to consider much larger supercell with a wide range of defect complexes forming in a V-4Ti alloy, including self-point defects (SPD), such as vacancies and self-interstitials (SIA).

Methods. Interatomic interactions are described using our newly developed interatomic potentials POT_TiV [6,7], which were fitted to DFT results and experimental data. The method is based on the Lipnitskii-Savelev form, which explicitly describes three-particle interactions and accounts for higher order interactions in the framework of the centrally symmetric approximation [8]. The POT_TiV potentials are consistent with diffusion properties, defect interactions, and melting temperature, which are essential for studying the mechanism governing radiation swelling reduction [7,9]. The elastic constants are correctly reproduced by the potentials, which is necessary for the correct description of the long-range strain fields around dislocations and other defect structures $[10,11]$. The potentials predicts that in pure $\mathrm{V}$ the vacancy dislocation loop $\langle 111\rangle$ is more energetically favorable than the void, consisting of the same number of vacancies, which is in qualitative agreement with experimental results obtained at low temperatures [12].

The MD simulations are performed using 2000-node bcc supercell for $\mathrm{V}$ and V-4Ti alloy with periodic boundary conditions. Ti atoms are randomly distributed around the V-4Ti cell, which is justified by the negligible Ti-Ti interaction of fewer than $0.06 \mathrm{eV}$ [5]. The visualization

\footnotetext{
* Corresponding author. Skolkovo Institute of Science and Technology, Moscow, Russia.

E-mail address: A.Boev@skoltech.ru (A.O. Boev).
} 
and analysis of the obtained structures are performed using the OVITO package [13]. The SIA position is defined as the coordinate of one atom in a [111] crowdion [9], which provide $99 \%$ detection accuracy due to temperature fluctuations (see Supplementary Section 2). The details of DFT calculations are provided in Supplementary Section 1.

Results and discussion. The formation energy of a self-point defect is univocally defined in a one-component atomic system. However, in an alloy, this energy depends on the local distribution of dopants. We calculate 1920 configurations of both the vacancy and SIA formation energies ( $E_{f}^{v}$ and $E_{f}^{S I A}$, respectively) in 2000-node supercell with random $4 \% \mathrm{Ti}$ distribution using our interatomic potentials. The distribution of $E_{f}^{v}$ and $E_{f}^{S I A}$ in V-4Ti, calculated using Eqs. S1 and S2 (Supplementary Section 1), is shown in Fig. 1. The vacancy formation energies are mostly centered around 2.1 and $1.6 \mathrm{eV}$, which corresponds to the formation of isolated and Ti-bound vacancy, respectively. The difference between these energies is equal to the binding energy of the Ti-Vac complex. $E_{f}^{v}$ has minimum values in a range of $1.1-1.3 \mathrm{eV}$ for a few number of configurations, which corresponds to the formation of different $2 \mathrm{Ti}-\mathrm{Vac}$ complexes. The SIA formation energies have three peaks: at $2.8 \mathrm{eV}$ from the $<111>$ crowdion, $2.6 \mathrm{eV}$ from the Ti-bound SIA, and the small, smeared $\sim 2.2 \mathrm{eV}$ peak from the 2Ti-SIA and 3Ti-SIA complexes. Thus, the Ti-SPD complexes have rich energy landscape, depending on the amount of Ti atoms (1-3) and their relative positions.

The atomic structure of the most favorable complexes and their binding energies calculated with POT_TiV and DFT are provided in Table 1 (the extended list is given in Supplementary Table S1). The binding energies calculated by POT_TiV are in good agreement with DFT results, despite their absence in the fitting procedure. This proves high transferability of the potentials required for quantitative study of the swelling reduction mechanism in a V-Ti system. The main ingredients for such mechanisms are the diffusion coefficients of SIA and vacancies in the presence of solutes, which are discussed further.

The diffusion coefficient for vacancies is calculated from the atomic square displacements (ASD), which was applied to all atoms as a function of temperature and for a fixed vacancy concentration [14]. The diffusion coefficient of SIA is calculated in two ways: from ASD for all atoms and from ASD only for atoms included in the SIA local atomic group (we will use the name $\mathrm{ASD}_{\text {loc }}$ ) [15]. We use 10 samples with a random start position of SPD and modeled them during $16 \mathrm{~ns}$ for every case.

The calculated vacancy and SIA diffusion coefficients in V and V-4Ti are plotted in Fig. 2 as a function of inverse temperature. According to Fig. 2 the activation energies for vacancy migration, determined by fitting to the Arrhenius equation in pure V and V-4Ti, are $0.45 \pm 0.01 \mathrm{eV}$ and $0.94 \pm 0.05 \mathrm{eV}$, respectively. The first value is consistent with the experimentally established values for vacancy migration of $0.5 \mathrm{eV}$ [16].

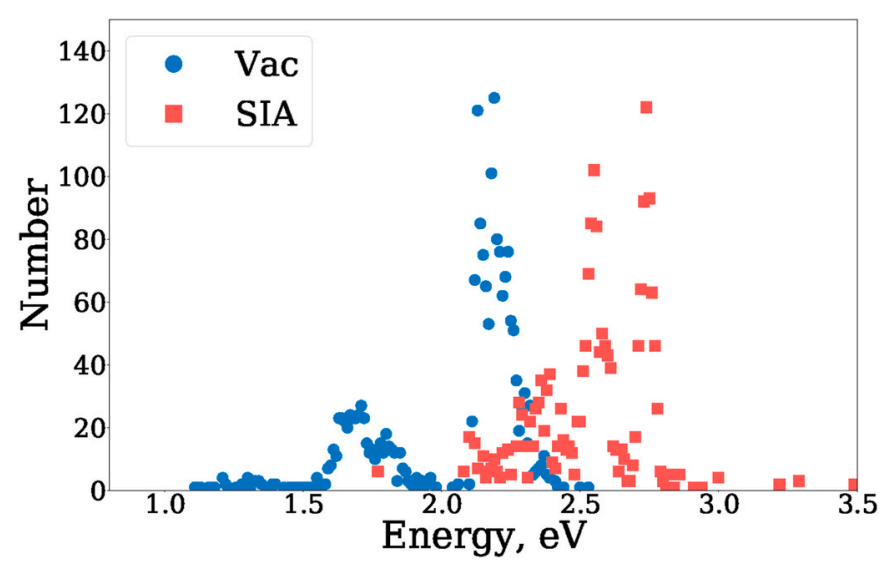

Fig. 1. Distribution of vacancy and SIA formation energies in a 2000 atom supercell of a V-4Ti random alloy.
Table 1

Binding energies of the main Ti-SPD complexes with respect to isolated point defects obtained in our previous works [5,7]. The dark spheres represent $\mathrm{Ti}$ atoms, while the dark rectangles represent vacancy positions. For the SIA complexes, the atomic projections are shown on the (111) plane with a crowdion in the center of the hexagon.

\begin{tabular}{llll}
\hline Complex & POT_TiV & DFT \\
\hline Ti-Vac & 0.50 & 0.37 \\
2Ti-Vac (No.5) & & 0.99 & 0.80 \\
Ti-SIA & & 0.21 & 0.15 \\
3Ti-SIA (No.3) & & & 0.35 \\
& & & 0.46 \\
\end{tabular}
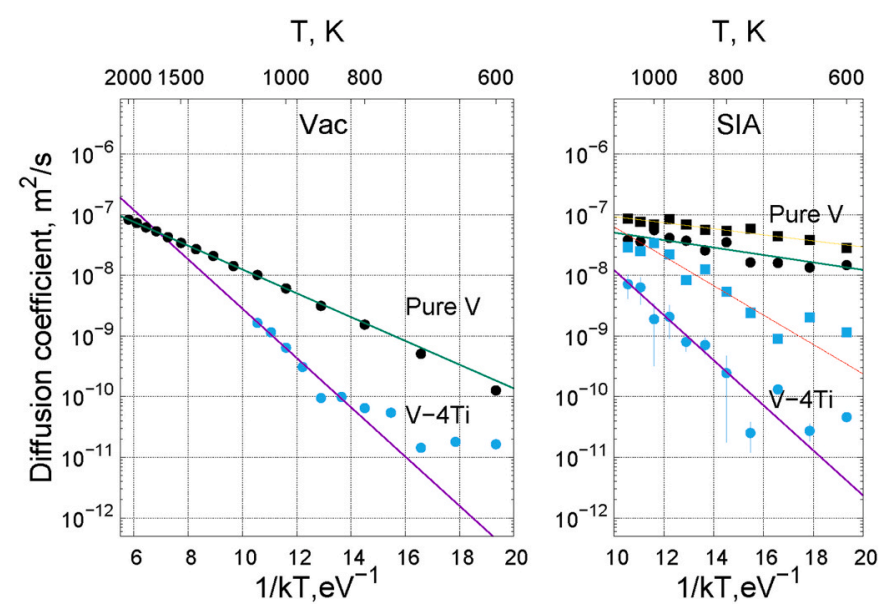

Fig. 2. Temperature dependence of vacancy and SIA diffusion coefficients for pure $\mathrm{V}$ and the V-4Ti alloy calculated using MD simulations with POT_TiV. SIA diffusion coefficients were calculated from ASD (circle dots) and from ASD $_{\text {loc }}$ (rectangular dots). For every case 10 samples with random SPD start position were modeled for $16 \mathrm{~ns}$.

In V-4Ti alloy vacancy migration energy is larger by a factor of 2, clearly showing that titanium atoms act as efficient vacancy traps. The $\sim 0.5 \mathrm{eV}$ difference between the migration energies in pure $\mathrm{V}$ and the alloy is consistent with the Ti-Vac binding energy, thereby confirming the assumption of the analytic model of swelling that vacancy trapping reduces its mobility. The activation energy for SIA diffusion in pure $\mathrm{V}$ is $0.14 \pm 0.04 \mathrm{eV}\left(0.11 \pm 0.01 \mathrm{eV}\right.$ obtained from $\left.\mathrm{ASD}_{\text {loc }}\right)$, which is consistent with a value $(0.14 \mathrm{eV})$ that was previously calculated by Minashin and Ryabov using MD simulations [17]. The migration energy of SIA in the V-4Ti alloy increases up to $0.85 \pm 0.14(0.56 \pm 0.09 \mathrm{eV}$ obtained from $\mathrm{ASD}_{\text {loc }}$ ), which is consistent with 2Ti-SIA binding energy $(0.3-0.5 \mathrm{eV})$ and 3Ti-SIA binding energy $(0.6-1.0 \mathrm{eV})$. The reduced SIA diffusion coefficient in the V-4Ti alloy is accompanied by a shorter migration pathway.

According to the theory of swelling the binding energy of SIA-solute must be higher than that of the vacancy-solute to produce comparable reduction of swelling: $E_{S I A}^{b} \sim E_{V a c}^{m}+E_{V a c}^{b}[18,19]$. In our case, the 
binding energy of Ti-SIA should be larger than $0.4 \mathrm{eV}$ to produce any swelling reduction and equal to $0.95 \mathrm{eV}$ to have the same effect as provided by Ti-Vac complexes. Comparing this with the calculated binding energies $(0.4-0.8 \mathrm{eV})$ of Ti-SIA complexes their contribution to swelling reduction should be of secondary importance.

After understanding the basic influence of Ti on the diffusion characteristics of the material, we investigate the evolution of excess vacancies formed after neutron bombardment. It is known from experiments and MD simulations that highly mobile SIAs move to the periphery in the cascade region, while monovacancies mostly stay in the center of the defective region [20-22].

We use three configurations of both 2000-atom V and V-4Ti samples $(30.3 \times 30.3 \times 30.3 \AA)$ with randomly distributed 20 and 50 monovacancies. Previously it was shown that $1 / 40$ th vacancy concentration is typical in the cascade region after neutron bombardment and cascade of atomic collisions [23,24]. The finish of evolution processes is tracked as a plateau of the potential energy with respect to supercell volume.

After NPT annealing over $16 \mathrm{~ns}$ at $700 \mathrm{~K}$, all vacancies in pure vanadium combine into one $1 / 2\langle 111\rangle$ Frank sessile dislocation loop (Supplementary Fig. S2). Such a dislocation loop is typical for bcc metals and was experimentally observed in pure vanadium after neutron irradiation [25,26]. Vacancies in the V-4Ti alloy maintain their individuality by forming Ti-vacancy complexes, as shown in Fig. 3. The analysis shows that these Ti-Vac structures contain up to 16 vacancies, although the minimum distance between vacancies is larger than or equal to the bcc lattice constant (2nd coordination sphere), preventing void formation. Recently, Lv et al. [27] reported a similar effect in the $\mathrm{Ta}-\mathrm{W}$ alloy, where $\mathrm{W}$ atoms suppress the aggregation of vacancy defects. The formation of Ti-Vac complexes aligns with the results from a positron annihilation study of a V-5Ti alloy, where no micropores were found except for monovacancies [28]. One example of such a complex obtained in our simulation is shown in Fig. 3. The same behaviour is obtained for smaller vacancy concentration of $1 / 100$. Final structures of $\mathrm{V}$ and V-4Ti samples with 20 and 50 vacancies after $16 \mathrm{~ns}$ at 700 and $1100 \mathrm{~K}$ are shown in Supplementary Fig. S2.

As a final part of our study, we evaluate possible SIA-vacancy recombination mechanisms for the dislocation loop and Ti-Vac microstructures. Although this particular simulation is not statistically robust, it allows us to see different behavior of SIA inside the area supersaturated by vacancies in $\mathrm{V}$ and $\mathrm{V}-4 \mathrm{Ti}$. In both microstructures, we consider ten configurations with different random initial positions of SIA, which migrates along the $\{111\}$ direction. MD annealing shows that SIA recombination with vacancies occurs only for half of the configurations in the case of pure $\mathrm{V}$ (with dislocation loop), where the mean free path of SIA exceeds the size of two supercells. Moreover, we find that recombination only occurs if the SIA path crosses over the dislocation loop. In the case of the V-4Ti alloy, SIA recombination occurs for every initial position with the mean free path less than the size of one supercell. An analysis of the migration trajectory shows that SIA is stuck in the defect zone, where it shuffles between the complexes and is unable to escape the zone until it recombines with one of the vacancies.

In conclusion, our MD simulation results show that titanium solutes reduce the mobility of vacancies, confirming the main assumption made in the analytic model of swelling. However, the reduction of mobility predicted by MD is smaller (Supplementary Fig. S3) than that by the analytic model [5]. More importantly, the MD implicitly demonstrates that radiation swelling is additionally suppressed by the formation of stable Ti-Vac complexes with up to 16 vacancies, preventing their association into pores or dislocation loops. In turn, such defective structure increases recombination rate with secondary SIA atoms, which are also trapped by the Ti solutes.

\section{Data availability statement}

The authors confirm that the data required to reproduce the findings of this study are available within the article and can be reproduced by

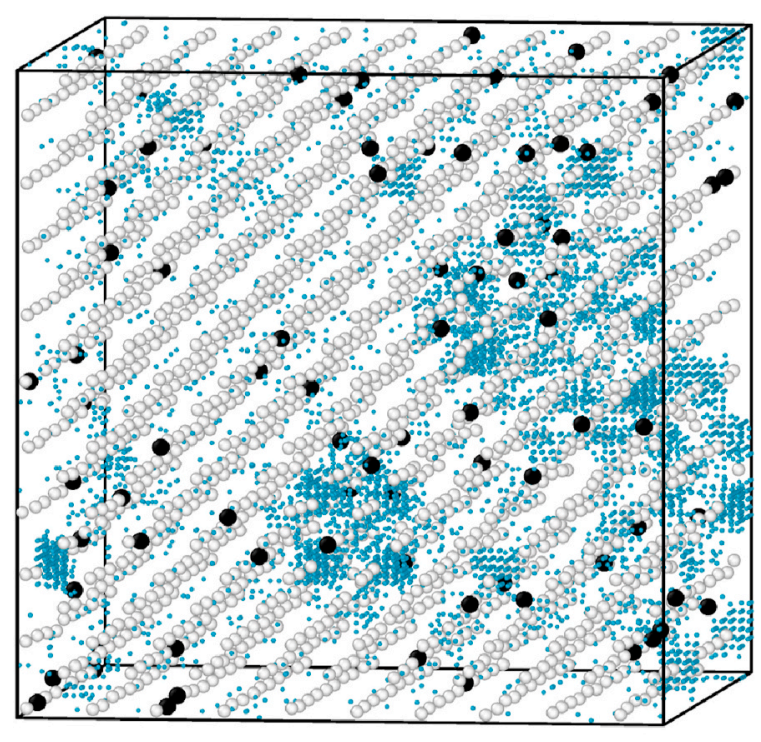

(100)

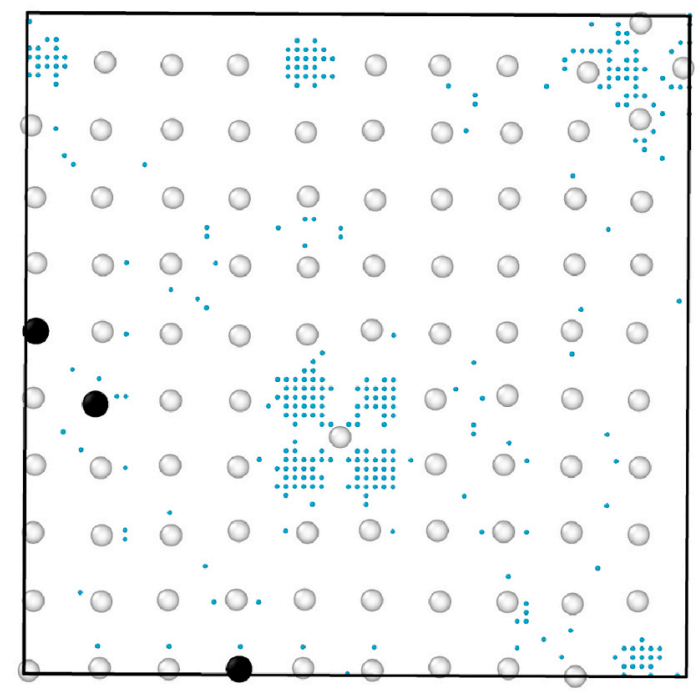

Ti-Vac complex
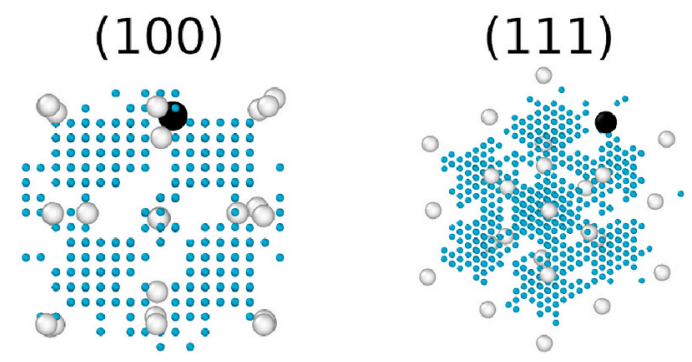

Fig. 3. 2000-node MD sample of V-4Ti after $16 \mathrm{~ns}$ of annealing at $700 \mathrm{~K}$. Additional view on the (100) plane of this sample. (100) and (111) projections of the Ti-Vac complex. Vacancies are shown with groups of small blue spheres determined using a method from the literature [29]; $\mathrm{V}$ and $\mathrm{Ti}$ atoms are designated with white and black spheres, respectively. 
molecular dynamic simulations.

\section{Declaration of competing interest}

The authors declare that they have no known competing financial interests or personal relationships that could have appeared to influence the work reported in this paper.

\section{Acknowledgments}

I.V. Nelasov acknowledges support from the State-supported research program for IPCP (No.AAAA-A19-119100800130-0) and A.G. Lipnitskii acknowledges support from RFBR grant No.18-02-00585 A.

\section{Appendix A. Supplementary data}

Supplementary data to this article can be found online at https://doi. org/10.1016/j.ssc.2021.114252.

\section{References}

[1] A. Impagnatiello, T. Toyama, E. Jimenez-Melero, J. Nucl. Mater. 485 (2017) $122-128$.

[2] D. Smith, M. Billone, K. Natesan, Int. J. Refract. Metals Hard Mater. 18 (2000) 213-224.

[3] I.R. Kirillov, In: Fundamentals of Magnetic Thermonuclear Reactor Design, Elsevier, 2018, pp. 291-321.

[4] B. Loomis, D. Smith, F. Garner, J. Nucl. Mater. 179 (1991) 771-774.

[5] A. Boev, D. Aksyonov, A. Kartamyshev, V. Maksimenko, I. Nelasov, A. Lipnitskii, J. Nucl. Mater. 492 (2017) 14-21.
[6] A. Kartamyshev, A. Lipnitskii, V. Saveliev, V. Maksimenko, I. Nelasov, D. Poletaev, Comput. Mater. Sci. 160 (2019) 30-41.

[7] A.I. Kartamyshev, A.G. Lipnitskii, A. Boev, I. Nelasov, V.N. Maksimenko, D. A. Aksyonov, T.K. Nguyen, Modelling and Simulation in Materials Science and Engineering, 2020.

[8] A. Lipnitskii, V. Saveliev, Comput. Mater. Sci. 121 (2016) 67-78.

[9] A. Boev, I. Nelasov, V. Maksimenko, A. Lipnitskii, V. Saveliev, A. Kartamyshev, Defect Diffusion Forum 375 (2017) 153-166.

[10] S. Dudarev, P.-W. Ma, Phys. Rev. Mater. 2 (2018), 033602.

[11] K. Tanimura, M. Fujiwara, T. Okada, T. Hagihara, Solid State Commun. 25 (1978) 315-318.

[12] Y. Candra, K. Fukumoto, A. Kimura, H. Matsui, J. Nucl. Mater. 271 (1999) 301-305.

[13] A. Stukowski, V.V. Bulatov, A. Arsenlis, Model. Simulat. Mater. Sci. Eng. 20 (2012), 085007.

[14] J.R. Manning, L. Bruner, Am. J. Phys. 36 (1968) 922-923.

[15] A. Boev, A. Lipnitskii, I. Nelasov, V. Saveliev, A. Kartamyshev, V. Maksimenko, K. Zolnikov, AIP Conf. Proc. 1909 (2017), 020018.

[16] P. Ehrhart, P. Jung, H. Schultz, Group III/25, Springer-Verlag, Berlin, 1991.

[17] A. Minashin, V. Ryabov, J. Nucl. Mater. 233 (1996) 996-998.

[18] L.K. Mansur, Nucl. Technol. 40 (1978) 5-34.

[19] S. Fisher, P. Madden, Phys. Status Solidi 69 (1982) 257-268.

[20] C. Woo, B. Singh, Phys. Status Solidi 159 (1990) 609-616.

[21] A.O. Boev, K.P. Zolnikov, I.V. Nelasov, A.G. Lipnitskii, Lett. Mater. 8 (2018) 263-267.

[22] A. Boev, K. Zolnikov, I. Nelasov, A. Lipnitskii, J. Phys, Conf. 1147 (2019), 012087.

[23] P. Brommer, L.K. Béland, J.-F. Joly, N. Mousseau, Phys. Rev. B 90 (2014) 134109.

[24] Y. Fan, A. Kushima, S. Yip, B. Yildiz, Phys. Rev. Lett. 106 (2011) 125501.

[25] H. Matsui, K. Fukumoto, D. Smith, H.M. Chung, W. Van Witzenburg, S. Votinov, J. Nucl. Mater. 233 (1996) 92-99.

[26] J. Gao, L. Cui, F. Wan, Mater. Char. 111 (2016) 1-7.

[27] Y. Lv, K. Hu, Z. Huang, Z. Cao, S. Wen, Y. Zhao, M. Pan, H. Deng, Solid State Commun. 306 (2020) 113767.

[28] T. Leguey, R. Pareja, E. Hodgson, J. Nucl. Mater. 231 (1996) 191-198.

[29] S.M. Davis, A.B. Belonoshko, B. Johansson, Comput. Phys. Commun. 182 (2011) 1105-1110. 